\title{
Identification of hub genes and pathways associated with bladder cancer based on co-expression network analysis
}

\author{
DONG-QING ZHANG, CHANG-KUO ZHOU, SHOU-ZHEN CHEN, YUE YANG and BEN-KANG SHI
}

Department of Urology, Qilu Hospital of Shandong University, Jinan, Shandong 250012, P.R. China

Received June 8,2015; Accepted February 27, 2017

DOI: $10.3892 / \mathrm{ol} .2017 .6267$

\begin{abstract}
The aim of the present study was to identify hub genes and signaling pathways associated with bladder cancer (BC) utilizing centrality analysis and pathway enrichment analysis. The differentially expressed genes (DEGs) were screened from the ArrayExpress database between normal subjects and BC patients. Co-expression networks of BC were constructed using differentially co-expressed genes and links, and hub genes were investigated by degree centrality analysis of co-expression networks in BC. The enriched signaling pathways were investigated by Kyoto Encyclopedia of Genes and Genomes database analysis based on the DEGs. The hub gene expression in BC tissues was validated using reverse transcription-quantitative polymerase chain reaction (RT-qPCR) and western blotting. A total of 329 DEGs were screened, including 147 upregulated and 182 downregulated genes. The co-expression network constructed between BC and normal controls consisted of 182 nodes and 434 edges, and the two genes in each gene pair were differentially co-expressed genes. Centrality analysis of co-expression networks suggested that the top 5 hub genes with high degree included lectin, galactoside-binding, soluble, 4 (LGALS4), protein tyrosine phosphatase, receptor type N2 (PTPRN2), transmembrane protease, serine 11E (TMPRSS11E), tripartite motif containing 31 (TRIM31) and potassium voltage-gated channel subfamily D member 3 (KCND3). Pathway analysis
\end{abstract}

Correspondence to: Dr Ben-Kang Shi, Department of Urology, Qilu Hospital of Shandong University, 107 Wenhua Xi Road, Jinan, Shandong 250012, P.R. China

E-mail: zhang68dq@163.com

Abbreviations: BC, bladder cancer; DEGs, differentially expressed genes; DCGL, differentially co-expressed genes; KEGG, Kyoto encyclopedia of genes and genomes; RT-qPCR, reverse transcription-quantitative polymerase chain reaction; DCGs, differentially co-expressed genes; DCLs, differentially co-expressed links; DCEA, differential co-expression analysis; DCp, differential co-expression profile; DCe, differential co-expression enrichment

Key words: bladder cancer, hub genes, co-expression network, pathway enrichment analysis, reverse transcription-quantitative polymerase chain reaction revealed that the 329 DEGs were significantly enriched in 5 terms (cell cycle, DNA replication, oocyte meiosis, p53 signaling pathway and peroxisome proliferator-activated receptor signaling pathway). According to RT-qPCR and western blot analysis, 4/5 hub genes were significantly expressed, including LGALS4, PTPRN2, TMPRSSIIE, TRIM31; however, KCND3 was not significantly expressed. In the present study, 5 hub genes were successfully identified (LGALS4, PTPRN2, TMPRSS11E, TRIM31 and KCND3) and 5 biological pathways that may be underlying biomarkers for early diagnosis and treatment associated with bladder cancer were revealed.

\section{Introduction}

Bladder cancer (BC) is the fourth most common cancer and the eighth leading cause of cancer-associated mortality worldwide. BC is an important public health issue as it is biologically very aggressive and highly prevalent in Western countries (1). In 2017, an estimated 79,030 new cases of BC and 16,870 mortalities will occur in the USA (2). In China, the incidence and mortality rates of $\mathrm{BC}$ rank the highest among tumors of the male urogenital system (3). Painless hematuria is the main symptom of $\mathrm{BC}$, and its diagnosis is established based on urinary cytology and transurethral tumor resection (4). There is an increasing trend in incidence and mortality rates of BC. Numerous studies have identified various risk factors that may induce $\mathrm{BC}$, including geography, race, gender, schistosomiasis infection, environmental or occupational exposure, smoking and genetic susceptibility (5-7).

Tumor progression is a complicated procedure of cancer cell development from normal epithelial cells, which involves changes in various genes, including oncogenes, cell cycle-associated genes, tumor suppressor genes and DNA damage repair genes. These are potential tumor markers in clinical practice; however, additional clinical studies are required to confirm their clinical utility (8-10). Development of molecular biology has increased the understanding of the mechanism underlying BC. Dyrskjøt et al (11) detected carcinoma in situ gene expression that is reflected in carcinoma in situ biopsies and superficial transitional cell carcinoma. Biton et al (12) demonstrated that a molecular urothelial differentiation program was maintained by applying independent component analysis to bladder cancer transcriptome data and exploiting additional molecular and clinic pathological data. 
Network-based approaches, particularly co-expression networks, offer a more effective means to identify potential malignancy diagnostic molecules based on connecting them together. Co-expression networks are generally used to study disease mechanisms (13) and provide a systems level view of dysregulated signaling pathways (14). The basic premise of co-expression analysis is that strongly correlated genes are likely to be functionally associated. Furthermore, it is possible to gain a clear insight into the important tumorigenic genes and signaling pathways of a variety of diseases, many of which are applicable to early detection and treatment (15).

In order to investigate the molecular and genetic mechanisms of $\mathrm{BC}$, the present study identified the differentially expressed genes (DEGs) between $\mathrm{BC}$ and normal controls and constructed co-expression networks of $\mathrm{BC}$ via differentially co-expressed genes and links (DCGL); subsequently, the hub genes and pathways were further investigated by centrality analysis and using the Kyoto Encyclopedia of Genes and Genomes (KEGG) database, respectively. In addition, the hub genes that were potential tumor markers for BC progression were validated in $\mathrm{BC}$ tissues using reverse transcription-quantitative polymerase chain reaction (RT-qPCR) and western blotting.

\section{Materials and methods}

Data collection and preprocessing. A total of two gene expression datasets from healthy people and $\mathrm{BC}$ patients, including E-MTAB-1940 and E-GEOD-3167, were obtained from the ArrayExpress database (www.ebi.ac.uk/arrayexpress/). A total of 146 samples (128 cases and 18 controls) were collected in the present study. The dataset E-MTAB-1940 (12) included 4 controls (samples from normal bladders) and 82 cases (samples from BC tissue); the dataset E-GEOD-3167 (11) included 14 controls and 46 cases.

Data preprocessing for all original expression information was performed prior to the analysis. In order to reduce the influence of nonspecific factors about, the background correction and normalization were performed using the robust multichip average method (16) and the quantile based algorithm (17), respectively. Perfect match and mismatch values were revised and selected using the Micro Array Suite 5.0 (MAS 5.0) algorithm (18) and the median method, respectively. Subsequently, the data were screened by the feature filter method of the genefilter package (http://bioconductor.org/packages/release/ bioc/html/genefilter.html). Each probe was mapped to one gene using getSYMBOL, whoch is is a function in package annotate of the genefilter package (http://www.bioconductor. org/packages/release/bioc/html/annotate.html), and the probe was discarded if it did not match any genes. The two expression datasets were merged and synthetically analyzed using Batch Mean-centering, a merged data method (19), following adaptation according to Support Vector Machines, through the inSilicoMerging package (20).

Detection of DEGs. In the present study, the DEGs between $\mathrm{BC}$ patients and normal controls were screened by the linear models for microarray data (LIMMA) package (21). $t$-tests and F-tests were performed on the matrix, and the P-values were transformed to $-\log 10$. Empirical Bayes (22) statistics and false discovery rate (23) calibration of P-values for the data were conducted using the $1 \mathrm{mFit}$ function (lmFit:http://lmfit.github. $\mathrm{io} / / \mathrm{lmfit}-\mathrm{py} /$ ). The DEGs were selected from following inspection for the following cut-off criteria: values of llog fold change (FC) $\mid \geq 2$ and $\mathrm{P}<0.05$.

Construction of differential co-expression networks by DCGL. It is critical to construct a co-expression network for identifying modules and the intra-modular connectivity. DCGL 2.0 (24) is an R package for identifying differentially co-expressed genes (DCGs) and differentially co-expressed links (DCLs) from gene expression microarray data. It examines the expression correlation based on the exact co-expression changes of gene pairs between two conditions, and thus is able to differentiate significant co-expression changes from relatively trivial ones (25). It has four functional modules: Gene filtration, link filtration, differential co-expression analysis (DCEA) and differential regulation analysis. Differential co-expression profile (DCp) and differential co-expression enrichment (DCe) are involved in the DCEA module for extracting DCGs and DCLs. DCp worked on the filtered set of gene co-expression value pairs, where each pair was composed of two co-expression values worked out under two different conditions separately $(24,26)$. The present study used a length-normalized Euclidean distance to measure differential co-expression (dC) of the co-expression value pairs associated with a particular gene. A permutation test was performed to assess the significance of $\mathrm{dC}$. The sample permutation was repeated $\mathrm{N}$ times, and a large number of permutation $\mathrm{dC}$ statistics formed an empirical null distribution. The P-value for each gene could then be estimated.

DCe was also used to identify DCGs and DCLs, which were based on the 'Limit Fold Change' (LFC) model. Initially, correlation pairs were divided into 3 parts according to the pairing of signs of co-expression values and the multitude of co-expression values: Pairs with the same signs, pairs with different signs and pairs with differently-signed high co-expression values. The first two groups were processed with the 'LFC' model separately to produce two subsets of DCLs, while the third group was added to the set of DCLs directly. Therefore, a total of DCLs was determined from all gene links.

Centrality analysis. To additionally assess the key genes of $\mathrm{BC}$, a centricity analysis was performed based on the nodes degree in the networks (27). Centrality measures mainly contain degree centrality, closeness centrality and shortest path between centrality, in which degree, the equivalent of the number of nodes directly adjacent to a given node (indicating the degree the vertex), is the simplest topological index (27). In the present study, centrality analysis, which was particularly useful to identify key players in biological processes, was implemented to study the differential co-expression networks. Calculation of the degree allows determining the 'degree distribution', which gives the probability that a selected node has exact links. Nodes with high degree (highly connected) are called 'hubs', which interact with several other genes, suggesting a central role in the interaction network (28). An obvious order of the vertices of a graph may be established by sorting them according to their degree (29). Genes with degree $\geq 9$ were defined as hub genes in the present study. 
Table I. Primer sequences and product length of the 5 hub genes.

\begin{tabular}{ll}
\hline Gene & \multicolumn{1}{c}{ Primer sequences (5'-3') } \\
\hline LGALS4 & Fength (bp) \\
& R: GGCTGCCACTTCAATCCGC \\
FTPRN2 & F: GTGGACCATGGAGTAGCTCG \\
& R: GTCCGAGAACCTCTCTGTCT \\
FMPRSS11E & F: GTCTCAGGATCGTTGGTGGG \\
& R: ACAAGAAAGTTGGCAAGATACCAGT \\
FRIM31 & F: GTCTTGTGCAGAAGTGAAGAGTT \\
& R: TCACAAAACCAAGCCCGGAT \\
KCND3 & F: TTTACACTGGAGGTGGGGGA \\
& R: TGCAGTGCGATTTCAGGTCT \\
Factin & F: AAGTACTCCGTGTGGATCGG \\
& R: TCAAGTTGGGGGACAAAAAG
\end{tabular}

LGALS4, lectin, galactoside-binding, soluble, 4; PTPRN2, protein tyrosine phosphatase, receptor type N2; TMPRSSI1E, transmembrane protease, serine 11E; TRIM31, tripartite motif containing 31; KCND3, potassium voltage-gated channel subfamily D member 3; F, forward; $\mathrm{R}$, reverse; bp, base pairs.

Pathway enrichment analysis. To investigate the enriched signaling pathways of the DEGs, enrichment analysis was performed based on the KEGG database (www.genome.jp/kegg/). The DEGs were applied to this database for investigating the association between the biochemical pathways and the occurrence of bladder cancer. The analysis was conducted by DAVID (30) (david.abcc.ncifcrf.gov/tools.jsp). The enrichment pathway analysis of the DEGs contributed to additional observation of these genes at the macroscopic level. Categories were obtained according to the Expression Analysis Systematic Explorer (31) score for 0.01 .

Patients. Tumor tissues from 10 BC patients were obtained during biopsy in Qilu Hospital of Shandong University (Jinan, China) between January 2015 and March 2015. Normal samples were collected from $2 \mathrm{~cm}$ away from the tumor. The present study was approved by the Ethical Committee of Qilu Hospital of Shandong University. Written informed consent was obtained from patients who agreed to participate in the study.

Validation of hub genes by RT-qPCR and western blotting. The tumor tissues of $10 \mathrm{BC}$ patients and normal tissue samples obtained from $2 \mathrm{~cm}$ away from the tumor were analyzed with polymorphic DNA markers, and total RNA was prepared using TRIzol (Beyotime Institute of Biotechnology- Haimen, China). Total RNA was reverse transcribed with an oligo (dT18) primer and was treated with $2 \mu \mathrm{l}$ RNasin $(40 \mathrm{U} / \mu \mathrm{l})$, $8.0 \mu \mathrm{l} 5 \mathrm{X}$ reverse transcriptase buffer, $8.0 \mu \mathrm{l}$ dNTPs and $2 \mu \mathrm{l}$ AMV reverse transcriptase $(5 \mathrm{U} / \mu \mathrm{l})$ according to the manufacturer's protocol. The RT reagents were purchased from Invitrogen (Thermo Fisher Scientific, Inc., Waltham, MA, USA). For RT-qPCR, primer sequences of the 5 hub genes are listed in Table I. The qPCR mix composition was as follows: $10 \mu \mathrm{l}$ of $10 \mathrm{X}$ qPCR buffer, $1 \mu \mathrm{l}$ of Taq DNA polymerase, $3 \mu 1$ of each forward and reverse primer and $8 \mu \mathrm{l}$ of dNTPs. The qPCR reagents were purchased from Invitrogen (Thermo
Fisher Scientific, Inc.). The qPCR conditions are shown in Table II. Complementary DNA was used as a template, and $\beta$-actin was used as an internal reference. RT-PCR products were separated using 1.5\% agarose gel electrophoresis and gels were visualized using Quantity One Software v4.62 (Bio-Rad Laboratories, Inc., Hercules, CA, USA). Every sample was run 3 times along with the internal control.

Proteins were extracted from tumor tissues and adjacent normal tissues according to the method reported by Yoon et al (32). Protein concentration was measured with a BCA Protein Assay kit (CW Biotech, Beijing, China). SDS-PAGE (12\%) was performed for $10 \mu \mathrm{g}$ protein, and the protein was electrotransferred $\left(4^{\circ} \mathrm{C}, 300 \mathrm{~mA}, 2 \mathrm{~h}\right)$ to a nitrocellulose membrane. The membrane was blocked in TBST containing $5 \%$ skimmed milk powder at $37^{\circ} \mathrm{C}$ for $2 \mathrm{~h}$, and incubated with rabbit anti-human antibodies $(1: 10,000)$ against lectin, galactoside-binding, soluble, 4 (LGALS4) [AP12391a; Abgent Biotech (SuZhou) Co., Ltd., Suzhou, China], protein tyrosine phosphatase, receptor type N2 (PTPRN2) (bs-19591R; BIOSS, Beijing, China), transmembrane protease, serine $11 \mathrm{E}$ (TMPRSS11E) [AP16520C; Abgent Biotech (SuZhou) Co., Ltd.], tripartite motif containing 31 (TRIM31) (bs-6220R; BIOSS), potassium voltage-gated channel subfamily D member 3 (KCND3) (bs-20219R; BIOSS) and GAPDH [AP50811; Abgent Biotech (SuZhou) Co., Ltd.] at $37^{\circ} \mathrm{C}$ for $2 \mathrm{~h}$. Unbound antibody was washed away by TBST ( 3 times), and the membrane was incubated with horseradish peroxdiase-labeled sheep anti-rabbit immunoglobulin $\mathrm{G}$ secondary antibody (1:5,000; SC-2048; Beijing Zhongshan Jinqiao Biotechnology Co., Ltd., Beijing, China) at $37^{\circ} \mathrm{C}$ for $2 \mathrm{~h}$. Following washing with TBST, the substrate was developed on the membrane for 3 min and exposed in the dark. Protein bands were visualized with Amersham ECL Western Blotting Detection kit (GE Healthcare Life Sciences, Chalfont, UK). GAPDH was used as an internal control, and western blots were analyzed using ImageJ 1.36b software (National Institutes of Health, Bethesda, MD, USA; http://rsb.info.nih.gov/ij/). 
Table II. Quantitative polymerase chain reaction amplification conditions for the 5 hub genes.

\begin{tabular}{lc}
\hline Gene & Reaction conditions \\
\hline LGALS4 & $94^{\circ} \mathrm{C} 1 \mathrm{~min} ; 35 \mathrm{cycles}$ of $98^{\circ} \mathrm{C} 10 \mathrm{sec}, 54^{\circ} \mathrm{C} 15 \mathrm{sec}, 68^{\circ} \mathrm{C} 1 \mathrm{~min} ; 72^{\circ} \mathrm{C} 7 \mathrm{~min}$ \\
PTPRN2 & $94^{\circ} \mathrm{C} 2 \mathrm{~min} ; 35 \mathrm{cycles}$ of $98^{\circ} \mathrm{C} 10 \mathrm{sec}, 50^{\circ} \mathrm{C} 15 \mathrm{sec}, 68^{\circ} \mathrm{C} 1 \mathrm{~min} ; 72^{\circ} \mathrm{C} 7 \mathrm{~min}$ \\
TMPRSSIIE & $94^{\circ} \mathrm{C} 2 \mathrm{~min} ; 33 \mathrm{cycles}$ of $98^{\circ} \mathrm{C} 10 \mathrm{sec}, 55^{\circ} \mathrm{C} 15 \mathrm{sec}, 68^{\circ} \mathrm{C} 1 \mathrm{~min} ; 72^{\circ} \mathrm{C} 7 \mathrm{~min}$ \\
TRIM31 & $94^{\circ} \mathrm{C} 2 \mathrm{~min} ; 35 \mathrm{cycles}$ of $98^{\circ} \mathrm{C} 10 \mathrm{sec}, 54^{\circ} \mathrm{C} 15 \mathrm{sec}, 68^{\circ} \mathrm{C} 1 \mathrm{~min} ; 72^{\circ} \mathrm{C} 7 \mathrm{~min}$ \\
KCND3 & $94^{\circ} \mathrm{C} 2 \mathrm{~min} ; 33 \mathrm{cycles}$ of $98^{\circ} \mathrm{C} 10 \mathrm{sec}, 54^{\circ} \mathrm{C} 15 \mathrm{sec}, 68^{\circ} \mathrm{C} 1 \mathrm{~min} ; 72^{\circ} \mathrm{C} 7 \mathrm{~min}$ \\
$\beta$-actin & $94^{\circ} \mathrm{C} 2 \mathrm{~min} ; 30 \mathrm{cycles}$ of $98^{\circ} \mathrm{C} 10 \mathrm{sec}, 51^{\circ} \mathrm{C} 15 \mathrm{sec}, 68^{\circ} \mathrm{C} 1 \mathrm{~min} ; 72^{\circ} \mathrm{C} 7 \mathrm{~min}$
\end{tabular}

LGALS4, lectin, galactoside-binding, soluble, 4; PTPRN2, protein tyrosine phosphatase, receptor type N2; TMPRSS11E, transmembrane protease, serine 11E; TRIM31, tripartite motif containing 31; KCND3, potassium voltage-gated channel subfamily D member 3.

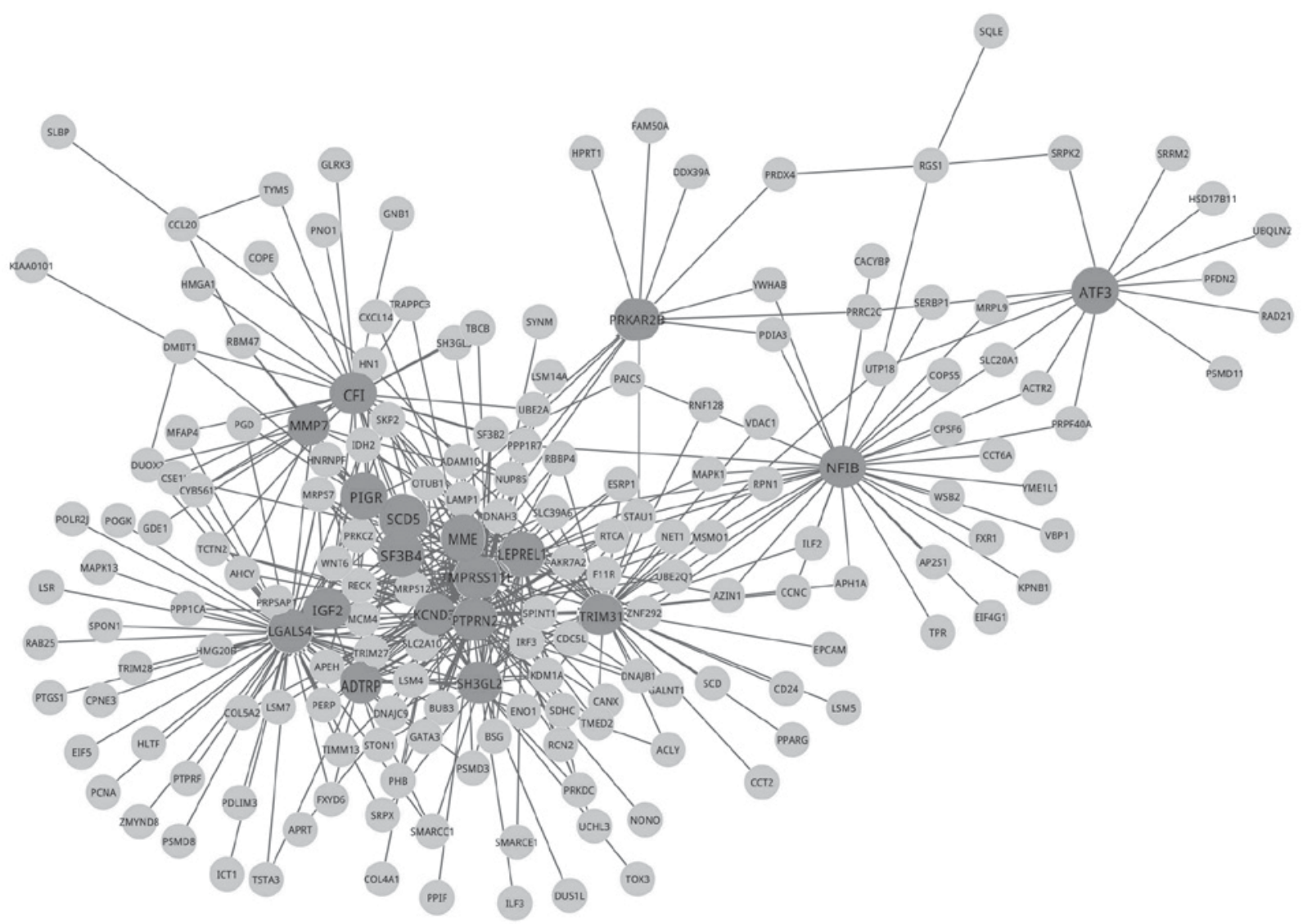

Figure 1. Co-expression network of bladder cancer based on 329 differentially expressed genes. There were 182 nodes and 43 edges in the co-expression network, where nodes referred to genes and edges between nodes indicated interaction of genes in the network; in particular, nodes in dark grey are hub genes.

Statistical analysis. Statistical analysis was conducted using SPSS 20.0 software (IBM SPSS, Armonk, NY, USA). All data were presented as mean \pm standard deviation. Statistical differences among groups were assessed using one way analysis of variance. $\mathrm{P}<0.05$ was considered to indicate a tatistically significanct difference. Graphs were designed using GraphPad Prism 5.0 (GraphPad Software, Inc., La Jolla, CA, USA).

\section{Results}

Detection of DEGs. In the present study, a total of 146 samples of two datasets associated with BC were preprocessed to iden- tify DEGs using the LIMMA package. A total of 329 DEGs, including 147 upregulated and 182 downregulated genes between $\mathrm{BC}$ patients and normal subjects were detected under the criteria of $\mid \operatorname{logFCl} \geq 2$ and $\mathrm{P}<0.05$.

Construction of differential co-expression networks by DCGL. The present study applied the DCGL 2.0 package in R to identify DCGs and DCLs, in which DCp and DCe methods involved in DCEA module were employed. A total of 434 DCLs were included in the co-expression network, and the two genes in each link were DCGs. A gene co-expression network containing 182 nodes and 434 edges was constructed in the analysis (Fig. 1). 
Table III. Eighteen hub genes where the degree was $\geq 9$ in the co-expression network.

\begin{tabular}{lc}
\hline Genes & Degree \\
\hline LGALS4 & 66 \\
PTPRN2 & 56 \\
TMPRSS1IE & 45 \\
TRIM31 & 39 \\
KCND3 & 35 \\
NFIB & 34 \\
LEPREL1 & 31 \\
SH3GL2 & 29 \\
CFI & 27 \\
ADTRP & 21 \\
PIGR & 16 \\
MME & 15 \\
ATF3 & 13 \\
MMP7 & 12 \\
PRKAR2B & 12 \\
SCD5 & 10 \\
IGF2 & 9 \\
SF3B4 & 9 \\
\hline
\end{tabular}

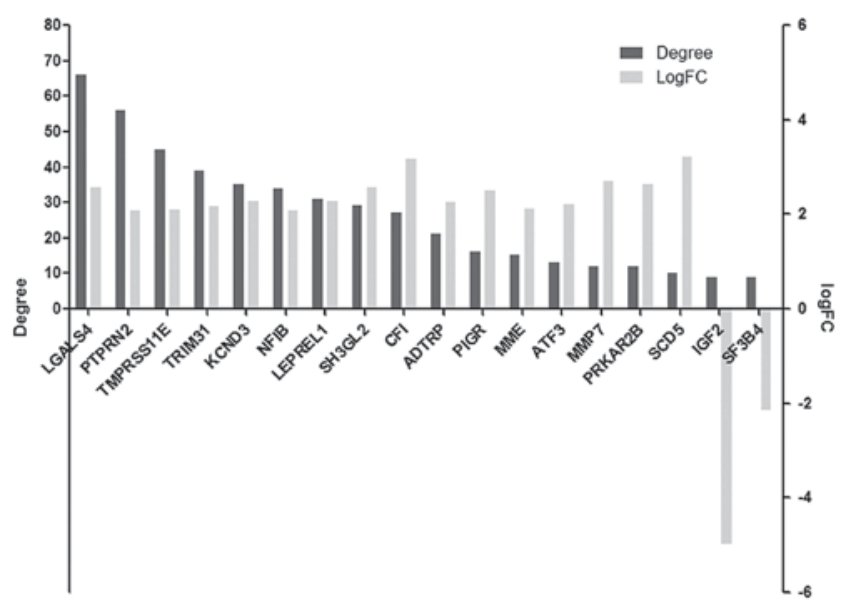

Figure 2. Summary of the degree value and $\log$ FC of the hub genes. FC, fold change.

Centrality analysis. By accessing degree centrality analysis of the co-expression network, 18 hub genes were obtained with degree $\geq 9$ (Table III), of which 16 genes were upregulated and 2 were downregulated (Fig. 2). The genes LGALS4, PTPRN2, TMPRSS11E, TRIM31 and KCND3 were the top 5 hub genes with high degree, and all of them were upregulated.

Pathway enrichment analysis. Pathway analysis based on the KEGG database revealed that these DEGs were significantly enriched in 5 terms, which were cell cycle $\left(\mathrm{P}=4.37 \times 10^{-7}\right)$, DNA replication $\left(\mathrm{P}=1.95 \times 10^{-3}\right)$, oocyte meiosis $\left(\mathrm{P}=6.47 \times 10^{-3}\right)$, p53 signaling pathway $\left(\mathrm{P}=7.17 \times 10^{-3}\right)$ and the peroxisome proliferator-activated receptor (PPAR) signaling pathway ( $\mathrm{P}=7.70 \mathrm{E}-03)$. Among the 5 terms, the cell cycle pathway was
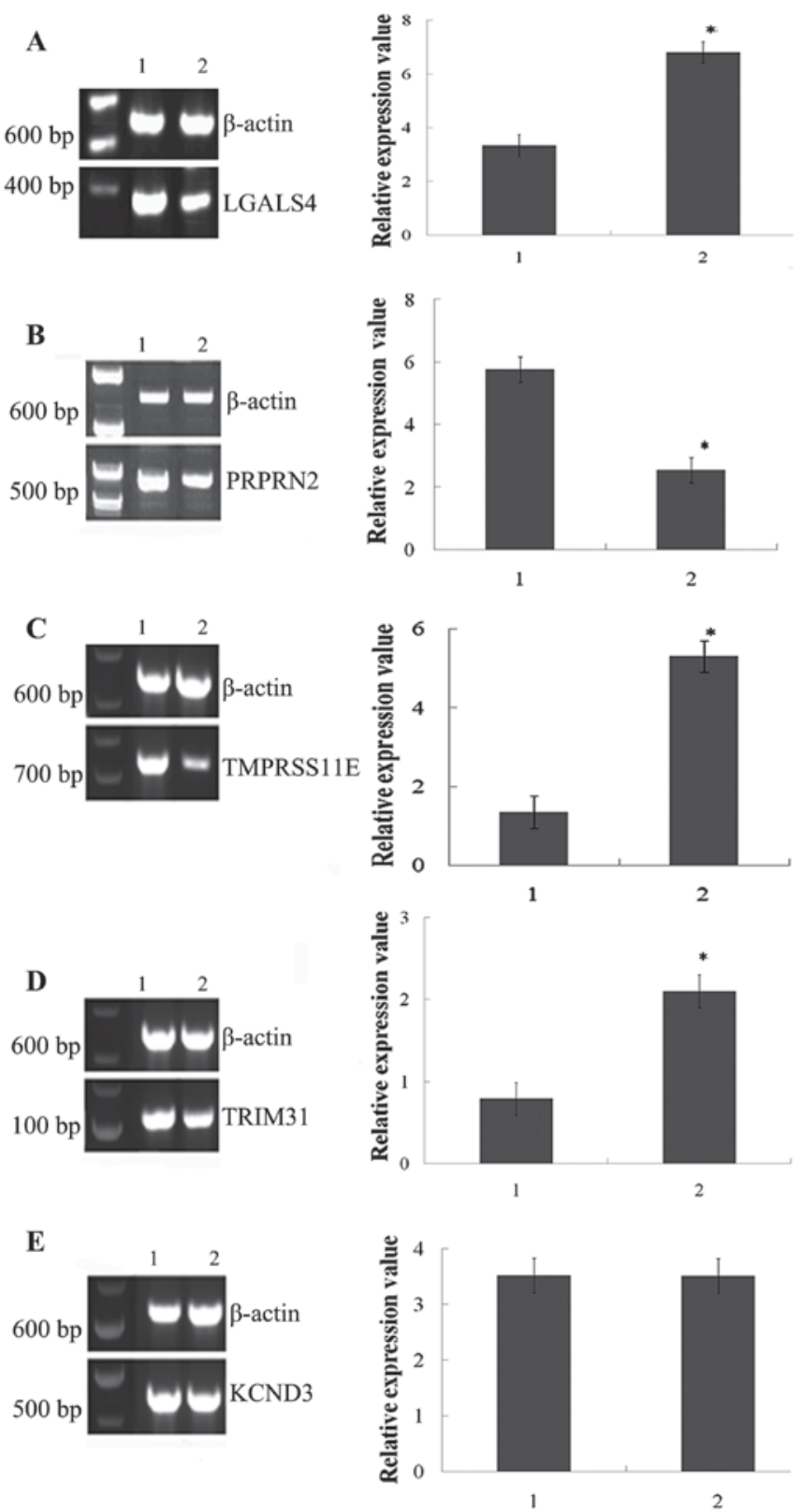

Figure 3. Results of reverse transcription-quantitative polymerase chain reaction analysis for 5 hub genes from the co-expression network. 1 represents the normal control and 2 represents disease. The following genes were investigated: (A) LGALS4, (B) PTPRN2, (C) TMPRSS11E, (D) TRIM31 and (E) KCND3. "P<0.05. LGALS4, lectin, galactoside-binding, soluble, 4; $P T P R N 2$, protein tyrosine phosphatase, receptor type N2; TMPRSS11E, transmembrane protease, serine 11E; TRIM31, tripartite motif containing 31 ; $K C N D 3$, potassium voltage-gated channel subfamily D member 3.

the most significant term, which also enriched more genes compared with the other terms.

Validation by RT-qPCR and western blotting. In the present study, to confirm the messenger RNA (mRNA) and protein expression levels of 5 hub genes (LGALS4, PTPRN2, TMPRSS11E, TRIM31 and KCND3) from the co-expression network, RT-qPCR and western blotting was performed on BC patient samples. The results of the relative expression of all the hub genes are shown in Figs. 3 and 4. It was observed that $4 / 5$ hub genes and the corresponding proteins including LGALS4, PTPRN2, TMPRSSIIE and TRIM31 were signifi- 
$\mathbf{A}$

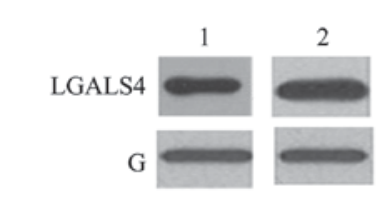

B

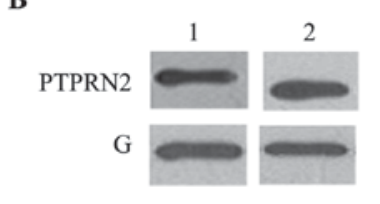

C

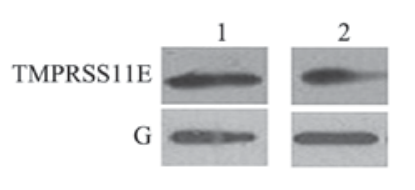

D

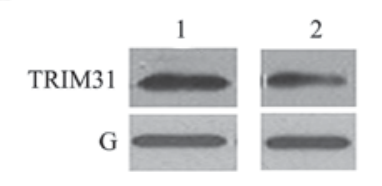

$\mathbf{E}$

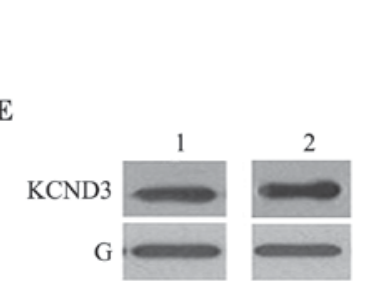

2

Figure 4. Results of western blot analysis for 5 hub genes. G represents GAPDH, 1 represents normal control and 2 represents disease. The following genes were analyzed: (A) LGALS4, (B) PTPRN2, (C) TMPRSS11E, (D) TRIM31 and (E) KCND3. " $\mathrm{P}<0.05$. LGALS4, lectin, galactoside-binding, soluble, 4; PTPRN2, protein tyrosine phosphatase, receptor type N2; TMPRSS11E, transmembrane protease, serine 11E; TRIM31, tripartite motif containing $31 ; K C N D 3$, potassium voltage-gated channel subfamily D member 3 .

cantly differentially expressed between $\mathrm{BC}$ patients and healthy subjects $(\mathrm{P}<0.05)$, while $K C N D 3$ was not significantly differentially expressed $(\mathrm{P}>0.05)$. Furthermore, it was noted that the relative expression of LGALS4, TMPRSSIIE and TRIM31 was increasing, which was consistent with the result of the bioinformatics analysis, while PTPRN2 demonstrated the opposite trend. $K C N D 3$, which was downregulated in the bioinformatics investigation, was not significantly differentially expressed.

\section{Discussion}

$\mathrm{BC}$ is a heterogeneous disease, with $30 \%$ of cases presenting as muscle-invasive disease associated with a high risk of death from distant metastases, which may be managed with transurethral resection (33). However, BC has a notable tendency to recur $(30-85 \%)$, therefore the present study investigated an adequate method for investigating biomarkers in $\mathrm{BC}$, that may contribute to our understanding of the pathogenesis and diagnoses of the disease, substantially reducing the mortality associated with this disease.

In the present study, hub genes and pathways of $\mathrm{BC}$ were identified based on degree centrality analysis of the co-expression network and pathway enrichment analysis. A total of 18 hub genes were obtained, the top 5 of which were LGALS4, PTPRN2, TMPRSS11E, TRIM31 and KCND3, by conducting centrality analysis on the co-expression network. Furthermore, cell cycle, DNA replication, oocyte meiosis, the p53 signaling pathway and the PPAR signaling pathway were observed to be significant pathways of BC. In addition, RT-qPCR and western blotting were performed to verify network-based results and investigate significant genes of BC.

RT-qPCR analysis revealed that 3 hub genes (LGALS4, TMPRSSIIE and TRIM31) were significantly upregulated in $\mathrm{BC}$ patients when compared with normal subjects, which was consistent with the bioinformatics analysis; however, KCND3 was not significantly differentially expressed between the conditions, and in contrast to the bioinformatics result, the relative content of the upregulated PTPRN2 was significantly reduced. This result was not entirely consistent with the network analysis. The probable reason for this was variations of samples; the microarray data was downloaded from the ArrayExpress database and RT-qPCR and western blotting were performed on patient samples. Therefore, it can be speculated that the 3 consistent hub genes (LGALS4, TMPRSS11E and TRIM31) may be potential markers of BC. LGALS4 has been identified as one of the genes involved in numerous types of human tumor, including sinonasal adenocarcinoma tumors (34), colorectal cancer (35) and breast cancer (36). There has also been a previous report that compared expression changes at mRNA and protein levels in the rat model and identified the gene exhibiting concordant changes with LGALS4 levels in bladder tumors (37). TRIM family proteins are involved in various cellular processes, including tumor development and antiviral response (38). One of the family proteins, TRIM31, was originally identified as a gene induced by growth-suppressive retinoid (39). A previous study showed that TRIM31 had the ability to regulate cell proliferation negatively in gastric adenocarcinoma (40) and its expression was reduced in lung cancer cell lines (41). However, the expression level of TRIM31 was increased in BC patients in the present study. Additional research is required to verify the biological properties of TRIM31.

Pathway analysis revealed that several significantly enriched signaling pathways included cell cycle, DNA replication, oocyte meiosis, the p53 signaling pathway and the PPAR signaling pathway. The cell cycle is the universal process through which cells reproduce and grow in all living organisms, and is concerned with the copying of the hereditary material, including replication of the chromosomal DNA during mitosis (42). Previous studies have indicated that several tumor suppressor candidates exert growth inhibitory effects by inducing cell cycle arrest at G2/M phase in leukemia cells (43), and exerted different tumor suppressive effects (44). 
In addition, Li et al (45) indicated the effect of the cell cycle on the susceptibility of SAS cells to sonodynamic therapy. p53 is a sequence-specific DNA-binding protein that promotes cell-cycle arrest or apoptosis in response to a variety of cellular stresses (46). The p53 signaling pathway had been suggested as a cellular surveillance mechanism for cancer prevention (47). Furthermore, drug development programs are underway to target the p53 signaling pathway (48).

In conclusion, the present study identified 5 hub genes associated with $\mathrm{BC}$, and 3 of these were verified via molecular experiments, RT-qPCR and western blotting. The signaling pathways associated with these genes were presented systematically. These genes and pathways may be potential biomarkers for early detection and therapy for BC.

\section{References}

1. Rubio G, García-Mora B, Santamaria C and Pontones JL: A flowgraph model for bladder carcinoma. Theor Biol Med Model 11 (Suppl 1): S3, 2014

2. Siegel RL, Miller KD and Jemal A: Cancer Statistics, 2017. CA Cancer J Clin 67: 7-30, 2017.

3. Chen WQ, Zeng HM, Zheng RS, Zhang SW and He J: Cancer incidence and mortality in China, 2007. Chin J Cancer Res 24 $1-8,2012$.

4. Sharma S, Ksheersagar P and Sharma P: Diagnosis and treatment of bladder cancer. Am Fam Physician 80: 717-723, 2009.

5. Burger M, Catto JW, Dalbagni G, Grossman HB, Herr H, Karakiewicz P, Kassouf W, Kiemeney LA, La Vecchia C, Shariat S and Lotan Y: Epidemiology and risk factors of urothelial bladder cancer. Eur Urol 63: 234-241, 2013.

6. Wyszynski A, Tanyos SA, Rees JR, Marsit CJ, Kelsey KT, Schned AR, Pendleton EM, Celaya MO, Zens MS, Karagas MR and Andrew AS: Body mass and smoking are modifiable risk factors for recurrent bladder cancer. Cancer 120: 408-414, 2014.

7. Letašiová S, Medve'ová A, Šovčíková A, Dušinská M, Volkovová K, Mosoiu C and Bartonová A: Bladder cancer, a review of the environmental risk factors. Environ Health 11 (Suppl 1): S11, 2012.

8. Xue Y, Wu G, Wang X, Zou X, Zhang G, Xiao R, Yuan Y, Long D, Yang J, Wu Y, et al: CIP2A is a predictor of survival and a novel therapeutic target in bladder urothelial cell carcinoma. Med Oncol 30: 406, 2013.

9. Tabata K, Matsumoto K, Minami S, Ishii D, Nishi M, Fujita T, Saegusa M, Sato Y and Iwamura M: Nestin is an independent predictor of cancer-specific survival after radical cystectomy in patients with urothelial carcinoma of the bladder. PLoS One 9: e91548, 2014.

10. Yan Y, Yang FQ, Zhang HM, Li J, Li W, Wang GC, Che JP, Zheng $\mathrm{JH}$ and Liu M: Bromodomain 4 protein is a predictor of survival for urothelial carcinoma of bladder. Int J Clin Exp Pathol 7: 4231-4238, 2014.

11. Dyrskjøt L, Kruhøffer M, Thykjaer T, Marcussen N, Jensen JL, Møller K and Ørntoft TF: Gene expression in the urinary bladder: A common carcinoma in situ gene expression signature exists disregarding histopathological classification. Cancer Res 64: 4040-4048, 2004.

12. Biton A, Bernard-Pierrot I, Lou Y, Krucker C, Chapeaublanc E, Rubio-Pérez C, López-Bigas N, Kamoun A, Neuzillet Y, Gestraud $\mathrm{P}$, et al: Independent component analysis uncovers the landscape of the bladder tumor transcriptome and reveals insights into luminal and basal subtypes. Cell Rep 9: 1235-1245, 2014

13. Nayak RR, Kearns M, Spielman RS and Cheung VG: Coexpression network based on natural variation in human gene expression reveals gene interactions and functions. Genome Res 19: 1953-1962, 2009.

14. Voineagu I, Wang X, Johnston P, Lowe JK, Tian Y, Horvath S, Mill J, Cantor RM, Blencowe BJ and Geschwind DH: Transcriptomic analysis of autistic brain reveals convergent molecular pathology. Nature 474: 380-384, 2011.

15. Kristensen VN, Lingjærde OC, Russnes HG, Vollan HK, Frigessi A and Børresen-Dale AL: Principles and methods of integrative genomic analyses in cancer. Nat Rev Cancer 14: 299-313, 2014
16. Ma L, Robinson LN and Towle HC: ChREBP*Mlx is the principal mediator of glucose-induced gene expression in the liver. J Biol Chem 281: 28721-28730, 2006.

17. Rifai $\mathrm{N}$ and Ridker PM: Proposed cardiovascular risk assessment algorithm using high-sensitivity C-reactive protein and lipid screening. Clin Chem 47: 28-30, 2001

18. Pepper SD, Saunders EK, Edwards LE, Wilson CL and Miller CJ: The utility of MAS5 expression summary and detection call algorithms. BMC Bioinformatics 8: 273, 2007.

19. Sims AH, Smethurst GJ, Hey Y, Okoniewski MJ, Pepper SD, Howell A, Miller CJ and Clarke RB: The removal of multiplicative, systematic bias allows integration of breast cancer gene expression datasets - improving meta-analysis and prediction of prognosis. BMC Med Genomics 1: 42, 2008.

20. Taminau J, Taminau MJ, Meganck S and BiocGenerics S: Package 'inSilicoMerging'. 2013. https://bioc.ism.ac.jp/packages/3.1/bioc/ $\mathrm{html} /$ inSilicoMerging.html. Access date, Feb 06, 2015.

21. Smyth G, Thorne N and Wettenhall J: LIMMA: Linear Models for Microarray Data User's Guide, 2003. http://www. bioconductor. org2005. Access date, Feb 08, 2015.

22. Datta S, Satten GA, Benos DJ, Xia J, Heslin MJ and Datta S: An empirical bayes adjustment to increase the sensitivity of detecting differentially expressed genes in microarray experiments. Bioinformatics 20: 235-242, 2004.

23. Reiner A, Yekutieli D and Benjamini Y: Identifying differentially expressed genes using false discovery rate controlling procedures. Bioinformatics 19: 368-375, 2003.

24. Yang J, Yu H, Liu BH, Zhao Z, Liu L, Ma LX, Li YX and Li YY: DCGL v2. 0: An R package for unveiling differential regulation from differential co-expression. PLoS One 8: e79729, 2013.

25. Yu H, Liu BH, Ye ZQ, Li C, Li YX and Li YY: Link-based quantitative methods to identify differentially coexpressed genes and gene pairs. BMC Bioinformatics 12: 315, 2011.

26. Liu BH, Yu H, Tu K, Li C, Li YX and Li YY: DCGL: An R package for identifying differentially coexpressed genes and links from gene expression microarray data. Bioinformatics 26: 2637-2638, 2010.

27. Scardoni G and Laudanna C: Centralities based analysis of complex networks. New Frontiers in Graph Theory. ISBN, 978-953-51-0115-4. INTECH Open Access Publisher, 2012 (http://www.intechopen.com/books/new-frontiers-ingraph-theory/centralities-based-analysis-of-networks).

28. Zotenko E, Mestre J, O'Leary DP and Przytycka TM: Why do hubs in the yeast protein interaction network tend to be essential: Reexamining the connection between the network topology and essentiality. PLoS Comput Biol 4: e1000140, 2008.

29. Koschützki D and Schreiber F: Centrality analysis methods for biological networks and their application to gene regulatory networks. Gene Regul Syst Bio 2: 193-201, 2008.

30. Huang DW, Sherman BT, Tan Q, Collins JR, Alvord WG, Roayaei J, Stephens R, Baseler MW, Lane HC and Lempicki RA: The DAVID gene functional classification tool: A novel biological module-centric algorithm to functionally analyze large gene lists. Genome Biol 8: R183, 2007.

31. Hosack DA, Dennis G Jr, Sherman BT, Lane HC and Lempicki RA: Identifying biological themes within lists of genes with EASE. Genome Biol 4: R70, 2003.

32. Yoon H and Liu RH: Effect of 2 alpha-hydroxyursolic acid on NF-kappaB activation induced by TNF-alpha in human breast cancer MCF-7 cells. J Agric Food Chem 56: 8412-8417, 2008.

33. Witjes JA, Compérat E, Cowan NC, De Santis M, Gakis G, Lebret T, Ribal MJ, Van der Heijden AG and Sherif A; European Association of Urology: EAU guidelines on muscle-invasive and metastatic bladder cancer: Summary of the 2013 guidelines. Eur Urol 65: 778-792, 2014.

34. Tripodi D, Quéméner S, Renaudin K, Ferron C, Malard O, Guisle-Marsollier I, Sébille-Rivain V, Verger C, Géraut C and Gratas-Rabbia-Ré C: Gene expression profiling in sinonasal adenocarcinoma. BMC Med Genomics 2: 65, 2009.

35. Selamat SA, Chung BS, Girard L, Zhang W, Zhang Y, Campan M, Siegmund KD, Koss MN, Hagen JA, Lam WL, et al: Genome-scale analysis of DNA methylation in lung adenocarcinoma and integration with mRNA expression. Genome Res 22: 1197-1211, 2012.

36. Miwa HE, Koba WR, Fine EJ, Giricz O, Kenny PA and Stanley P: Bisected, complex N-glycans and galectins in mouse mammary tumor progression and human breast cancer. Glycobiology 23 : 1477-1490, 2013

37. Lu Y, Liu P, Wen W, Grubbs CJ, Townsend RR, Malone JP, Lubet RA and You M: Cross-species comparison of orthologous gene expression in human bladder cancer and carcinogen-induced rodent models. Am J Transl Res 3: 8-27, 2010. 
38. Hatakeyama S: TRIM proteins and cancer. Nat Rev Cancer 11: 792-804, 2011.

39. Dokmanovic M, Chang BD, Fang J and Roninson IB: Retinoid-induced growth arrest of breast carcinoma cells involves co-activation of multiple growth-inhibitory genes. Cancer Biol Ther 1: 24-27, 2002.

40. Sugiura T and Miyamoto K: Characterization of TRIM31, upregulated in gastric adenocarcinoma, as a novel RBCC protein. J Cell Biochem 105: 1081-1091, 2008.

41. Li H, Zhang Y, Zhang Y, Bai X, Peng Y and He P: TRIM31 is downregulated in non-small cell lung cancer and serves as a potential tumor suppressor. Tumour Biol 35: 5747-5752, 2014.

42. Nurse P: A long twentieth century of the cell cycle and beyond. Cell 100: 71-78, 2000

43. Peng ZG, Yao YB, Yang J, Tang YL and Huang X: Mangiferin induces cell cycle arrest at G2/M phase through ATR-Chk1 pathway in HL-60 leukemia cells. Genet Mol Res 14: 4989-5002, 2015.

44. Andries V, Vandepoele K, Staes K, Berx G, Bogaert P, Van Isterdael G, Ginneberge D, Parthoens E, Vandenbussche J, Gevaert K and van Roy F: NBPF1, a tumor suppressor candidate in neuroblastoma, exerts growth inhibitory effects by inducing a G1 cell cycle arrest. BMC Cancer 15: 391, 2015.
45. Li N, Sun M, Wang Y, Lv Y, Hu Z, Cao W, Zheng J and Jiao X: Effect of cell cycle phase on the sensitivity of SAS cells to sonodynamic therapy using low-intensity ultrasound combined with 5-aminolevulinic acid in vitro. Mol Med Rep 12: 3177-3183, 2015. 46. de Stanchina E, McCurrach ME, Zindy F, Shieh SY, Ferbeyre G, Samuelson AV, Prives C, Roussel MF, Sherr CJ and Lowe SW: E1A signaling to p53 involves the p19ARFtumor suppressor. Genes Dev 12: 2434-2442, 1998.

47. Chakraborty A, Uechi T and Kenmochi N: Guarding the 'translation apparatus': Defective ribosome biogenesis and the p53 signaling pathway. Wiley Interdiscip Rev RNA 2: 507-522, 2011.

48. Stegh AH: Targeting the p53 signaling pathway in cancer therapy - the promises, challenges and perils. Expert Opin Ther Targets 16: 67-83, 2012. 